\title{
Geovisualisierung zur räumlichen Entscheidungsunterstützung
}

\author{
Claus Rinner \\ Ryerson University
}

digital.library.ryerson.ca/object/203

Please Cite:

Rinner, C. (2007). Geovisualisierung zur räumlichen

entscheidungsunterstützung. [Geovisualization to support spatial decisionmaking]. Kartographische Nachrichten, 2, 85-92.

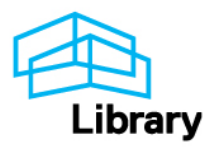




\title{
Geovisualisierung zur räumlichen Entscheidungsunterstützung
}

\author{
Claus Rinner, Toronto, Kanada
}

In diesem Beitrag wird die Kombination der räumlichen mehrkriteriellen Bewertung mit Prinzipien der Geovisualisierung vorgestellt. Durch die interaktive Manipulation von Kartensymbolik, durch verknüpfte Displays sowie transiente Selektion und Hervorhebung von Datenpunkten können mehrkriterielle Entscheidungsstrategien flexibel durchgespielt werden.

Die Ergebnisse unterschiedlicher Szenarien können dann miteinander verglichen und mit dem Sachwissen des Benutzers abgestimmt werden. Zwei Fallstudien für die Nutzung der Geovisualisierung zur räumlichen Entscheidungsunterstützung in der Gesundheitsvorsorge und zur Abschätzung der urbanen Lebensqualität werden vorgestellt. Durch die kartenzentrierte, interaktive Exploration von Kriterienhierarchien und -gewichten können tiefere Einblicke in diese Entscheidungsprobleme gewonnen werden, als es mit herkömmlichen Methoden möglich wäre.

- Schlüsselbegriffe: Geovisualisierung, Entscheidungsstrategien, Gesundheitsvorsorge, Lebensqualität

This paper presents a combination of methods for spatial multi-criteria evaluation with principles of geographic visualization. Decision-makers are enabled to test multi-criteria decision strategies through the interactive manipulation of map symbols, linked displays, and transient selection and highlighting of data points. The results of different scenarios can then be compared and calibrated with the user's knowledge. The paper introduces two case studies for geographic visualization in spatial decision support for public health planning and urban quality of life assessment. The case studies illustrate how map-centred interactive exploration of criteria hierarchies and importance weights affords a deeper insight into decision problems than traditional methods.

Keywords: geographic visualization, decision strategies, public health planning, quality of life

\section{Einleitung}

Geographische Informationssysteme (GIS) werden häufig implizit als räumliche Entscheidungsunterstützungssysteme (spatial decision support systems - SDSS) benutzt oder dienen explizit als Entwicklungsplattformen für SDSS (Keenan, 1997). Densham (1991) bezeichnet die Modellbasis als eine wichtige Komponente von SDSS. Eine besondere Art von mathematischen Modellen, die in SDSS Anwendung findet, sind Verfahren zur mehrkriteriellen Bewertung (multi-criteria evaluation - MCE). Seit Anfang der 1990er-Jahre sind MCE-Verfahren in GIS implementiert und zur räumlichen Entscheidungsfindung in verschiedensten Anwendungsfeldern genutzt worden (Malczewski, 1999).

Während Karten in SDSS traditionell als Bestandteil der Berichterstellung gelten, ergeben sich durch die dynamische Entwicklung der Geovisualisierung neue Rollen für interaktive Karten in der räumlichen Entscheidungsunterstützung. So wurden in den vergangenen Jahren einige Verfahren zur iterativen Festlegung 
von mehrkriteriellen Einstellungen und zur räumlich-visuellen Exploration der Zwischenergebnisse vorgeschlagen. Diese kartenzentrierte Herangehensweise an die räumliche MCE ist das Thema dieses Beitrags.

Im Weiteren wird zunächst die Entwicklung der traditionellen MCE in GIS zusammengefasst. Anschließend werden die Grundzüge der Geovisualisierung sowie ihre Anwendung auf die räumliche Entscheidungsunterstützung vorgestellt. In zwei praktischen Anwendungen zur Bewertung des Gesundheitsstands der Bevölkerung der Provinz Ontario, Kanada, sowie der Lebensqualität von Stadtvierteln in der Metropole Toronto, Kanada wird die kartenzentrierte MCE illustriert. Im Schlussabschnitt werden Ergebnisse und weiterer Forschungsbedarf diskutiert.

\section{Mehrkriterielle Bewertung in der räumlichen Entschei- dungsunterstützung}

Die mehrkriterielle Bewertung (auch: „multikriterielle Analyse”) ist ein einfaches Berechnungsverfahren aus dem Bereich der Betriebswirtschaftslehre, mit dessen Hilfe eine Reihe alternativer Lösungswege in eine Rangfolge gebracht wird. Diese Rangfolge kann dann als Entscheidungshilfe verstanden werden. Ein Vorteil der MCE ist die Integration einer Reihe von Entscheidungskriterien in eine einheitliche Bewertungszahl unter Berücksichtigung des Ausgleichs schlechter Leistungen in manchen Kriterien durch gute Leistungen in anderen. Derartige "kompensatorische" Methoden stehen im Gegensatz zum „harten” (booleschen) Filtern der Alternativen, bei dem alle Kriterien gleichermaßen erfüllt werden müssen.

Aufgrund ihrer typischen Komplexität sind räumliche Entscheidungsprobleme wie beispielsweise die Suche nach einem Standort für eine industrielle Anlage oder Entsorgungseinrichtung in der Regel durch viele mögliche Entscheidungskriterien gekennzeichnet, die zumindest teilweise gegeneinander ausgeglichen wer-
KN 2/2007 den können. Daher hat es seit Anfang

der 1990er-Jahre eine Reihe von Studien gegeben, in denen MCE-Verfahren in GIS implementiert wurden (Janssen \& Rietveld, 1990; Carver, 1991; Church et al., 1992; Banai, 1993; Pereira \& Duckstein, 1993, Jankowski, 1995; Eastman, 1997; siehe auch die Zusammenfassung in Malczewski, 1999). Dabei wurden verschiedene GIS und Verknüpfungsansätze sowie diverse Anwendungsfälle beschrieben. In einem Review-Artikel subsumiert Malczewski (2006) die-typische MCEMethoden und -Anwendungen im Bereich GIS. Von mehr als 300 dahingehend untersuchten wissenschaftlichen Arbeiten sind beispielsweise bei $26 \%$ die Komponenten MCE und GIS nicht miteinander integriert, bei $33 \%$ liegt eine lockere Verknüpfung vor, bei $30 \%$ eine enge Verknüpfung, und lediglich $11 \%$ sind voll integrierte Ansätze. Bezüglich der Anwendungsdomänen GIS-basierter MCE ragt Umweltschutz/Ökologie mit $17 \%$ der untersuchten Arbeiten heraus, gefolgt von Verkehr, Stadtplanung, Abfallmanagement, Hydrologie, Landwirtschaft und Forstwirtschaft mit $11 \%$ bis $8 \%$ (Malczewski, 2006).

Gemeinsam ist diesen Ansätzen, dass das Entscheidungsproblem in einer Matrix beschrieben werden kann, deren Zeilen die Lösungsalternativen als geographische Standorte (Punkte, Polygone oder Rasterzellen) repräsentieren und deren Spalten die Entscheidungskriterien widerspiegeln. Die Matrix enthält die Leistung (performance) der einzelnen Standorte in allen Kriterien, die nach einer Standardisierung numerisch vergleichbar gemacht und zeilenweise zu einer einzigen Bewertungszahl zusammengefasst werden. Die Bewertungszahl ermöglicht den Vergleich der Standorte und - im einfachsten Fall - die Auswahl des am besten bewerteten.

Der Raumbezug in der räumlichen MCE lässt sich zunächst einmal an der geographischen Ausprägung der Entscheidungsalternativen festmachen, deren Kriterienwerte in der Regel als Attribute räumlicher Objekte in GIS-Datenbanken 
zugänglich sind. Dadurch ist die Darstellung der Alternativen, ihrer Bewertung und letztendlich die der getroffenen Entscheidung auf Karten möglich. In den vergangenen Jahren sind zusätzlich auch Arbeiten begonnen worden, die weitere, speziell räumliche Aspekte der MCE behandeln. So haben van Herwijnen \& Rietveld (1999) erstmals räumlich inhomogene Entscheidungsziele (objectives) und Kriteriengewichte zugelassen. Feick \& Hall (2004) untersuchen die räumliche Dimension der Sensitivität der Bewertungsergebnisse auf Änderungen in den Entscheidungskriterien. Rinner \& Heppleston (2006) schlagen eine Systematisierung räumlich definierter Entscheidungskriterien nach den zugrundeliegenden räumlichen Relationen (Ort, Nähe und Richtung) vor und experimentieren mit einer Post-Hoc-Anpassung der resultierenden Bewertungszahlen aufgrund räumlicher Gruppierungen der alternativen Standorte.

\section{Kartographische Visualisierung räumlicher Entscheidungsstrategien}

McCormick et al. (1987) haben den Begriff der "Visualisierung" für flexible graphische Darstellungen großer wissenschaftlicher Datensätze geprägt, bei deren Erkundung die mächtige Bildverarbeitungsfähigkeit des Menschen genutzt werden kann. In Bezug auf räumliche Datensätze sind zwar schon immer Karten als graphische Hilfsmittel eingesetzt worden, die Rolle von Karten im wissenschaftlichen Prozess hat sich jedoch mit dem Aufkommen der Geovisualisierung Anfang der 1990er-Jahre dramatisch geändert.

Monmonier (1989) stellt die Methode des geographic brushing vor, die auf einer dynamischen Verknüpfung einer thematischen Karte mit Streudiagrammen basiert. MacEachren \& Ganter (1990) beschreiben den grundsätzlichen neuen Ansatz der Visualisierung räumlicher Daten mit dem Ziel der Datenexploration und Entdeckung räumlicher Muster. DiBiase
KN 2/2007 (1990) hat die Rolle von Karten im wissenschaftlichen Prozess als ein Medium zur Datenexploration und Hypothesenbestätigung in der Frühphase einer Analyse und als Ergänzung zur gängigen Rolle von Karten zur Kommunikation von Forschungsergebnissen neu definiert. Dieses Modell wurde von MacEachren (1994) zum Würfelmodell der Kartennutzung ausgeweitet, das im wesentlichen feststellt, dass Karten entlang einer Achse von Kommunikation zu Visualisierung genutzt werden und verstärkt durch interaktive Funktionalität der Entdeckung von Neuem in einem privaten (Forschungs-)Umfeld dienen.

Slocum et al. (2005) stellen eine Reihe von Software-Werkzeugen zur Geovisualisierung vor, die jedoch weitgehend noch im Stadium von Forschungsprototypen verharren. Insbesondere werden die Grenzen von kommerziellen GIS in Hinblick auf die unmittelbare Systemantwort auf Nutzereingaben diskutiert. Im Gegensatz dazu ist beispielsweise das Forschungssystem CommonGIS (Andrienko \& Andrienko, 1999) dadurch gekennzeichnet, dass die Manipulation kartographischer Variablen mit dem Ziel der Datenexploration sich unmittelbar auf die Bildschirmkarte auswirkt und dass alle geöffneten Karten und Graphen dynamisch miteinander verknüpft sind.

Dieses System wurde von Jankowski et al. (2001) im Zusammenhang mit räumlicher Entscheidungsunterstützung eingesetzt. Die Autoren stellen die Nutzung interaktiver Karten zur Bearbeitung einer mehrkriteriellen Analyse mit Hilfe des Ideal-Point-Verfahrens dar. Die visuelle Herangehensweise unterstützt hier die Strukturierung des Entscheidungsproblems. Andrienko \& Andrienko (2001; 2003) stellen weitere visuelle Entscheidungsunterstützungswerkzeuge in CommonGIS vor, z. B. interaktive Slider zur Manipulation der Kriteriengewichte und Utility-Symbole zur vergleichenden Darstellung von standardisierten Kriterienwerten.

Der Ablauf eines kartenbasierten MCEProzesses ist in Abb. 1 schematisch dar- 
gestellt. Ausgehend von einer als gegeben angenommenen Menge machbarer (feasible) Alternativen und von feststehenden Nutzerpräferenzen wird die mehrkriterielle Bewertung anhand einer Entscheidungsstrategie vorgenommen. Im Falle der in diesem Artikel verwendeten Bewertungsmethoden beinhaltet diese Strategie die Auswahl von Entscheidungskriterien, die Anordnung der Kriterien in einer Hierarchie, die Festlegung von Bedeutungsgewichten für die Kriterien und gegebenenfalls die Festlegung eines zweiten Gewichtevektors. Die resultierende Rangfolge wird geographisch visualisiert und somit die Entscheidungsstrategie einer Exploration und Revision unterzogen, die auf dem Expertenwissen des Analysten basiert.

Rinner \& Taranu (2006) haben die gängige MCE-Methode analytic hierarchy process (AHP) auf der Geovisualisierungsplattform CommonGIS implementiert und anhand einer Analyse des Gesundheitsstands der Bevölkerung der kanadischen Provinz Ontario getestet. Diese Arbeit wird im folgenden Abschnitt 4 zusammengefasst. Rinner \& Malczewski (2002) und Malczewski \& Rinner (2005) haben eine Familie von MCE-Methoden, das ordered weighted averaging (OWA), zu den Entscheidungsunterstützungsmethoden in CommonGIS hinzugefügt und auf die Bewertung der Lebensqualität in verschiedenen Stadtbereichen in London, Ontario, angewandt, wie in Abschnitt 5 beschrieben.

\section{Fallstudie 1: Bewertung des Gesundheitsstands der Bevölkerung Ontarios}

Die öffentliche Gesundheit (public health) ist ein vielschichtiges Konzept, das in aller Regel über die bloße Abwesenheit von Erkrankungen hinaus die Lebensqualität der Bevölkerung widerspiegelt. Medizinische, demographische und sozio-ökonomische Faktoren werden zur Abschätzung des Gesundheitsstands der Bevölkerung herangezogen. In den Studien von Birch et al. (2000), Irbarren et al. (2001),

KN 2/2007 Kosteniuk \& Dickinson (2003) und Wen

3632 et al. (2003) wurden Zusammenhänge zwischen Einkommen, Bildungsstand und Erwerbstätigkeit auf der einen Seite und öffentlicher Gesundheit auf der anderen Seite nachgewiesen. Goel et al. (1996) diskutieren solche Gesundheitsindikatoren einerseits individuell, fordern jedoch andererseits zusammenfassende Maßzahlen für die öffentliche Gesundheit.

Rinner \& Taranu (2006) schlagen vor, mehrkriterielle Verfahren zur Erstellung einer einheitlichen Bewertungszahl für den Gesundheitsstand administrativer Gebiete (public health units) zu nutzen. Die verwendeten Gesundheitsindikatoren sind nicht-medizinische Variablen aus einer Erhebung von Statistics Canada (2006), wie in Tab. 1 aufgezeigt. Da die in Tab. 1 wiedergegebenen Variablen bereits in einer fachlichen Hierarchie eingeordnet sind, bietet sich unter den verfügbaren MCE-Methoden der Analytic Hierarchy Process (AHP) an. Dieses von Saaty (1980) entwickelte Verfahren vereinfacht mehrkriterielle Entscheidungsprobleme dadurch, dass Entscheidungskriterien in einer Hierarchie angeordnet werden und die Festlegung der Kriteriengewichte auf den verschiedenen Hierarchie-Ebenen separat erfolgt. Weiterhin hat Saaty (1980) den paarweisen Vergleich von Kriterien eingeführt, durch den die Kriteriengewichtung innerhalb der Hierarchie-Ebenen weiter vereinfacht wird.

In Rinner \& Taranu (2006) wurde das AHP-Verfahren in dem interaktiven thematischen Kartographie-Werkzeug CommonGIS (Voss et al., 2000) implementiert. Abb. 2 zeigt die Benutzeroberfläche zur Festlegung der AHP-Hierarchie und zum paarweisen Kriterienvergleich. Durch die Verknüpfung von Methoden der MCE mit denen der Geovisualisierung wird es möglich, verschiedene Entscheidungsstrategien auf visuellem Weg zu erkunden. Prinzipien der Geovisualisierung werden beispielsweise angewandt, wenn die Karte mit Bewertungszahlen bei jeder Änderung der MCE-Parameter unmittelbar aktualisiert wird. Ein Beispiel für die Explo- 
ration unterschiedlicher Entscheidungsstrategien ist in Abb. 3 gegeben.

Die in Abb. 3 dargestellte Änderung der Rangfolge der bewerteten Gebiete basiert auf einer sukzessiven Entwicklung einer Kriterien-Hierarchie mittels des AHP-Verfahrens. In Abb. 3a sind alle 13 Kriterien gleichgewichtet (keine Hierarchie). In Abb. 3b wurde die in Tab. 1 dargestellte Hierarchie der nicht medizinischen Gesundheitsfaktoren umgesetzt. Untergruppen und Kriterien innerhalb übergeordneter Gruppen sind jeweils gleichgewichtet. In Abb. 3c wurden die Kriteriengewichte innerhalb dieser Hierarchie manuell angepasst. Beispielsweise erhielten die beiden ersten Kategorien auf der obersten Hierarchie-Ebene, gesundheitsrelevantes Verhalten und Lebens- und Arbeitsumstände, jeweils 40 \% Gewicht, während persönliche Ressourcen und Umweltfaktoren nur mit jeweils $10 \%$ gewichtet wurden. Die Auswirkungen der unterschiedlichen Gewichtungen sind beispielsweise an den public health units im Norden Ontarios deutlich erkennbar.

\section{Fallstudie 2: Bewertung der Lebensqualität in London, Ontario}

Sozio-ökonomische und demographische Faktoren spielen auch bei der Bewertung der urbanen Lebensqualität in Städten eine herausragende Rolle. Traditionell wurden wohlhabende Gebiete mit hohem Bildungsstand und geringer Arbeitslosenrate als kennzeichnend für eine hohe Lebensqualität gesehen (GC, 1978; FCM, 1999). In jüngerer Zeit werden Arbeitsplätze im künstlerisch-kreativen Bereich und die Diversität der Herkunft (Anteil Einwanderer), der sexuellen Orientierung (Anteil gleichgeschlechtlicher Partnerschaften) und der Unterkunftsarten (Anteil Mietwohnungen) als für die urbane Lebensqualität positive Faktoren interpretiert (Florida, 2002; Gertler et al., 2002). Die Mehrzahl der Studien zur Lebensqualität untersucht Unterschiede zwischen Städten oder zwischen Ländern

KN 2/2007 und die daraus resultierenden Wande-

3632

5 rungsbewegungen hin zu den attraktivsten Zentren. Ferner werden meist die betrachteten Faktoren einzeln diskutiert. Massam (2002) stellt dagegen eine Verbindung zwischen einer integrierten Bewertung von Lebensqualität und mehrkriteriellen EntscheidungsunterstützungsMethoden her. Malczewski \& Rinner (2005) nutzen MCE zur integrierten Abschätzung der Lebensqualität von Nachbarschaften innerhalb der Stadt London, Ontario. Diese Anwendung soll im Weiteren beschrieben werden.

Tab. 2 zeigt die Liste der in dieser Fallstudie berücksichtigten sozio-demographischen Variablen, die einer klassischen Sicht auf urbane Lebensqualität entsprechen. Diese Variablen wurden in einem integrierten Ansatz als Entscheidungskriterien zu einer Bewertungszahl für jedes Stadtviertel (statistischer Bezirk) kombiniert.

Die mehrkriterielle Methode für diese Kombination ist das ordered weighted averaging (OWA, nach Yager, 1988). OWA beschreibt eine Familie von mehrkriteriellen Operatoren, die die gewichtete Summer (simple additive weighting, oder auch weighted linear combination) mathematisch verallgemeinern. Neben den Kriteriengewichten erfordert OWA die Festlegung weiterer Gewichtungsfaktoren, der order weights. Nach der Multiplikation der Kriteriengewichte w[i] mit den standardisierten Kriterienwerten $a^{\prime}[i]$ jeder Entscheidungsalternative werden die Terme $w[i] \cdot a^{\prime}[i]=: b[i]$ der Größe nach absteigend sortiert. Die order weights v[j] werden nun auf die b[j] angewandt, um das obere oder untere Ende der sortierten Folge zu verstärken. In einer optimistischen Sichtweise könnten Entscheidungsträger beispielsweise die besten Werte jeder Alternative verstärken. In einer pessimistischen Herangehensweise dagegen würden die schlechtesten Werte betont. Nach Anwendung der order weights werden die Terme $v[j] \cdot b[j]$ aufsummiert und ergeben die abschließende Bewertungszahl für jede Alternative. 
Abb. 4 zeigt die CommonGIS-basierte Benutzeroberfläche zur Festlegung der order weights und die daraus resultierende Entscheidungsstrategie. Die zwei Dimensionen im dreieckigen Raum der OWA-Entscheidungsstrategien sind Risiko (risk) und Zielkonflikt (tradeoff). Das Entscheidungsrisiko ist hier als die Nähe zur optimistischen Strategie definiert. Der Zielkonflikt beschreibt das Ausmaß, in dem schlechte Werte in einem Kriterium durch gute Werte in einem anderen ausgeglichen werden können.

Abb. 5 verdeutlicht, dass trotz einer grundlegenden Änderung der Entscheidungsstrategie von moderat optimistisch über neutral bis moderat pessimistisch einige Stadtbezirke im Norden und Westen Londons durchweg unter den besten zehn Gebieten liegen. Damit können Entscheidungsalternativen identifiziert werden, die unter verschiedenen Strategien als konsensfähig erscheinen.

\section{Ergebnisse und Ausblick}

In diesem Artikel wurden zwei Fallstudien vorgestellt, die anhand zweier mehrkriterieller Bewertungsmethoden die Nutzung der Geovisualisierung zur Exploration von Entscheidungsstrategien demonstrieren. Es kann festgestellt werden, dass die Bereitstellung interaktiver Karten von Zwischenergebnissen einer Bewertung die Erarbeitung einer idealen Strategie unterstützt. In der AHP-Methode beinhaltet die Entscheidungsstrategie die Auswahl von Kriterien und deren Anordnung in einer Hierarchie sowie die Festlegung von Kriteriengewichten. Im Falle der OWAMethode schlagen sich die Nutzerpräferenzen in der Auswahl der Kriterien, deren Gewichtung und der Festlegung von Entscheidungsrisiko und Zielkonflikt mittels eines weiteren Gewichtevektors nieder.

Das Durchspielen zahlreicher Parametersetzungen kann als Sensitivitätsanalyse bezüglich der Entscheidungsstrategien verstanden werden; die Auswirkungen kleiner, manueller Veränderungen der $\mathrm{Pa}$ rameter werden untersucht. Das Augen-
KN 2/2007 merk des Entscheidungsträgers wird da-
3632

6 mit auf die unter verschiedenen Strategien stabil bewerteten Gebiete sowie auf mögliche Ursachen für instabile Bewertungen gelenkt. Durch den Abgleich mit vorhandenem räumlichen Wissen kann der kartenbasierte Entscheidungsprozess zu einem besseren Verständnis des Entscheidungsproblems und letztlich zu besseren Entscheidungen führen.

Aktuelle Forschungsprojekte befassen sich mit der Interpretation weiterer Aspekte von MCE, insbesondere die Standardisierung der Kriterien, als Bestandteile von Entscheidungsstrategien. Hierfür liegen unterschiedliche Methoden vor (u. a. lineare Skalierung, Min-max-Transformation), deren unterschiedliche Ergebnisse mittels Geovisualisierung exploriert werden könnten. Ferner sollten weitere gängige MCE-Verfahren sowie weitere Entscheidungsunterstützungsfunktionen (z. B. ein Report-Generator) auf derselben Analyse-Plattform (z. B. CommonGIS) implementiert werden, um dem Benutzer ein umfassendes SDSS anzubieten.

Anhand der Anwendung zur urbanen Lebensqualität werden zurzeit Tests der Zweckmäßigkeit der entwickelten Methoden und der Benutzerfreundlichkeit ihrer Implementation durchgeführt, die Aufschluss über die Anwendbarkeit in realen Entscheidungssituationen geben können. In Bezug auf die räumlich-visuelle Analyse im Gesundheitswesen hat bereits eine Kooperation mit einer Heilfürsorgeeinrichtung begonnen, im Rahmen derer die Anwender eine Entscheidung für einen neuen Standort in einem besonders bedürftigen Gebiet getroffen haben. Weitere Test-Anwendungen sind geplant, um die Möglichkeiten und Grenzen der beschriebenen Methoden zu untersuchen.

\section{Dank}

Die hier vorgestellten Forschungsergebnisse basieren auf Kooperationen mit Jacek Malczewski und John Taranu, denen mein Dank gebührt. Von Martin Rapp stammen hilfreiche Korrekturen am 
Manuskript. Das Natural Sciences and Engineering Research Council of Canada (NSERC) hat diese Arbeiten teilweise finanziell unterstützt.

\section{Literatur}

Andrienko, G., Andrienko, N. (1999): Interactive Maps for Visual Data Exploration. International Journal Geographical Information Science 13: 355-374

Andrienko, N., Andrienko, G. (2001): Intelligent Support for Geographic Data Analysis and Decision Making in the Web. Journal of Geographic Information and Decision Analysis 5(2): 115-128

Andrienko, N., Andrienko, G. (2003): Informed Spatial Decisions through Coordinated Views. Information Visualization 24: 270-85

Banai, R. (1993): Fuzziness in Geographic Information Systems: Contributions from the Analytic Hierarchy Process. International Journal of Geographical Information Systems 7: 315-329

Birch, S., Jerrett, M., Eyles, J. (2000): Heterogeneity in the determinants of health and illness: the example of socio-economic status and smoking. Social Science and Medicine 51(2): 307-317

Carver S. J. (1991): Integrating Multi-Criteria Evaluation with Geographical Information Systems. International Journal of Geographical Information Systems 5(3): 321-339

Church R. L., Loban S. R., Lombard K. (1992): An Interface for Exploring Spatial Alternatives for a Corridor Location Problem. Computers and Geosciences 8: 1095-1105

Densham, P.J. (1991): Spatial Decision Support Systems. In: D. J. Maguire, M. F. Goodchild, D. W. Rhind (eds.) Geographical Information Systems: Principles and Applications. Longman, London, pp. 403-412

DiBiase, D. (1990): Visualization in the Earth Sciences. Earth and Mineral Sciences, Bulletin of the College of Earth and Mineral Sciences, Pennsylvania State University, 59(2): 13-18

Eastman, J. R. (1997): IDRISI for Windows, Version 2.0: Tutorial Exercises. Graduate School of Geography, Clark University, Worcester, MA

FCM (Federation of Canadian Municipalities) (1999): The FCM Quality of Life Reporting System: Quality of Life in Canadian Communities. Ottawa

Feick, R., Hall, G. B. (2004): A Method for Examining the Spatial Dimension of Multi-criteria Weight Sensitivity. International Journal of Geographical Information Science 18(8): 815-840

Florida, R. (2002): The Economic Geography of Talent. Annals of the Association of American Geographers 92(4):743-755

GC (Government of Canada) (1978) Urban Indicators: Statistical Profiles of Quality of Life for Canadian Cities. Revised edition, Ministry of State of Urban Affairs, Ottawa

Gertler, M. S., Florida, R., Gates, G., and Vinodrai, T. (2002): Competing on Creativity: Placing Ontario's
KN 2/2007 for the Ontario Ministry of Enterprise, Opportunity and Innovation and the Institute for Competitiveness and Prosperity, November 2002. Online unter http://www.utoronto.ca/progris/Competing\%20on\%20 Creativity\%20in\%200ntario\%20Report\%20(Nov\%20 22).pdf [14 May 2006]

Goel, V., Iron, K., Williams, I. (1996): Indicators of Health Determinants and Health Status. In Goel, V., Williams, J. I., Anderson, G. M., Blackstien-Hirsch, P., Fooks, C., Naylor, C.D. (eds) Patterns of Health Care in Ontario. The ICES Practice Atlas, 2nd edition, Ottawa, Canadian Medical Association. Online unter http://www.ices.on.ca/ [26 January 2006]

van Herwijnen, M., Rietveld, P. (1999): Spatial Dimensions in Multicriteria Analysis. In: Thill, J.-C. (ed.) Spatial Multicriteria Decision Making and Analysis: A Geographic Information Sciences Approach. Ashgate, New York, pp. 77-99

Irbarren, C., Friedman, G.D., Klatsky, A. L., Eisner, M.D. (2001): Exposure to environmental tobacco smoke: association with personal characteristics and self reported health conditions. Journal of Epidemiology and Community Health 55(10): 721-728

Jankowski, P. (1995): Integrating Geographical Information Systems and Multiple Criteria Decision Making Methods. International Journal of Geographical Information Systems 9: 251-273

Jankowski, P., Andrienko, N., Andrienko, G. (2001): Map-Centered Exploratory Approach to Multiple Criteria Spatial Decision Making. International Journal of Geographical Information Science 15: 101-127

Janssen R., Rietveld P. (1990): Multicriteria Analysis and Geographical Information Systems: An Application to Agricultural Land Use in the Netherlands. In: Scholten HJ, Stillwell JCH (eds.) Geographical Information Systems for Urban and Regional Planning. Kluwer, Dordrecht, pp 129-139

Keenan, P. (1997): Using a GIS as a DSS Generator. University College Dublin, Department of Management Information Systems, Working Paper MIS 95-9. Online unter http://mis.ucd.ie/staff/pkeenan/ gis_as_a_dss.html [05 September 2006]

Kosteniuk, J. G., Dickinson, H.D. (2003): Tracing the social gradient in the health of Canadians: primary and secondary determinants. Social Science and Medicine 57(2): 263-276

MacEachren, A. M., Ganter, J. H. (1990): A Pattern Identification Approach to Cartographic Visualization. Cartographica 27(2): 64-81

MacEachren, A. M. (1994): Visualization in Modern Cartography: Setting the Agenda. In A. M. MacEachren, D. R. F. Taylor, Visualization in Modern Cartography. Pergamon, Oxford, UK

Malczewski, J. (1999): GIS and Multicriteria Decision Analysis. New York: John Wiley \& Sons

Malczewski, J., Rinner, C. (2005): Exploring Multicriteria Decision Strategies in GIS with Linguistic Quantifiers: A Case Study of Residential Quality Evaluation. Journal of Geographical Systems 7(2): 249-268

Malczewski, J. (2006): GIS-Based Multicriteria Decision Analysis: A Survey of the Literature. International 
Journal of Geographical Information Science 20(7): 703-726

Massam, B.H. (2002): Quality of life: public planning and private living. Progress in Planning 58(3): 141-227.

McCormick, B. H., DeFanti, T.A., Brown, M.D. (eds.) (1987) Visualization in Scientific Computing. Computer Graphics 21(6)

Monmonier, M. (1989) Geographic Brushing: Enhancing Exploratory Analysis of the Scatterplot Matrix. Geographical Analysis 21: 81-84

Pereira, J. M. C., Duckstein, L. (1993) A Multiple Criteria Decision-Making Approach to GIS-Based Land Suitability Evaluation. International Journal of Geographical Information Systems 7: 407-424

Poetz, A. (2003): Spatial Patterns of Residential Burglaries in London, Ontario. Unveröffentlichte B.A. Thesis, University of Western Ontario, London, Ontario

Rinner, C., Malczewski, J. (2002): Web-Enabled Spatial Decision Analysis Using Ordered Weighted Averaging (OWA). Journal of Geographical Systems 4(4): 385-403

Rinner, C., Taranu, J. (2006): Map-Based Exploratory Evaluation of Non-Medical Determinants of Population Health. Transactions in GIS 10(4): 633-649

Rinner, C., Heppleston, A. (2006): The Spatial Dimensions of Multi-Criteria Evaluation - Case Study of a Home Buyerß Spatial Decision Support System. In M. Raubal, H. Miller, A. Frank, M. Goodchild (eds.) Geographic Information Science - Fourth International Conference, GIScience 2006, Münster, Germany, September 2006. Lecture Notes in Computer Science, Volume 4197. Springer, Berlin

Saaty, T. L. (1980): The Analytic Hierarchy Process. McGraw-Hill, New York

Slocum, T., McMaster, R. B., Kessler, F. C., Howard, H. H. (2005): Thematic Cartography and Geographic Visualization. Second Edition, Prentice Hall, Upper Saddle River, NJ

Statistics Canada (2006): Health Indicators, Vol. 2006 No. 1. Online unter http://www.statcan.ca/english/ freepub/82-221-XIE/82-221-XIE2006001.htm [05 September 2006]

Statistics Canada (1996): Census of Population, 1996. Datenzugriff über das Internet Data Library System, University of Western Ontario, London, Ontario

Voss, H., Andrienko, N., Andrienko, G. (2000): CommonGIS: Common Access to Geographically Referenced Data. ERCIM News 41(4): 44-6. Online unter http://www.ercim.org/publication/Ercim_News/enw41/ voss.html [30 August 2006]

Wen, M., Browning, C.R., Cagney, K.A. (2003): Poverty, affluence, and income inequality: neighborhood economic structure and its implications for health. Social Science \& Medicine 57(5): 843-860

Yager, R.R. (1988): On ordered weighted averaging aggregation operators in multi-criteria decision making. IEEE Transactions on Systems, Man and Cybernetics 18(1): 183-190
KN 2/2007

3632

Anschrift des Verfassers: Dr. Claus Rinner, Assistant Professor, Department of Geography, Ryerson University, 350 Victoria Street, Toronto ON M5B 2K3, Canada; E-Mail: crinner@ryerson.ca 


\title{
KN 2/2007
}

3632

\begin{abstract}
Abb. 1:
Ablaufdiagramm für iterative ParameterFestlegung/-Änderung und (geo)graphischer Exploration der (neuen) Zwischenergebnisse mit Abgleich gegen Nutzerwissen
\end{abstract}

Abb. 2: Die Benutzeroberfläche zur Festlegung der AHP-Hierarchie und zum paarweisen Kriterienvergleich

Abb. 3:

Rangfolge der public health units

in Ontario unter verschiedenen AHPEinstellungen: a) Alle 13 Kriterien gleichgewichtet,

keine Hierarchie.

b) Kriterien inner-

halb hierarchischer

Gruppierung gleichgewichtet.

c) Manuelle Gewich-

tung innerhalb der

Kriterien-Hierarchie.

Abb. 4: Die Benutzeroberfläche zur Festlegung der order weights in der OWA-Methode und Anzeige der resultierenden Entscheidungsstrategie

Abb. 5: Ergebnis

der Bewertung der

Lebensqualität nach

verschiedenen

Entscheidungs-

strategien.

a) Moderat

optimistische,

b) neutrale, und

c) moderat pessimis-

tische Strategie.

Tab. 1: Ausgewählte nicht-medizinische Gesundheitsfaktoren (Datenquelle: Statistics Canada, 2006) und ihre Bewertungsrichtung

Tab. 2: Liste der sozio-demographischen Variablen zur Bewertung der Lebensqualität in London, Ontario und ihre Bewertungsrichtung (Quelle: Statistics Canada, 1996; Poetz, 2003) 


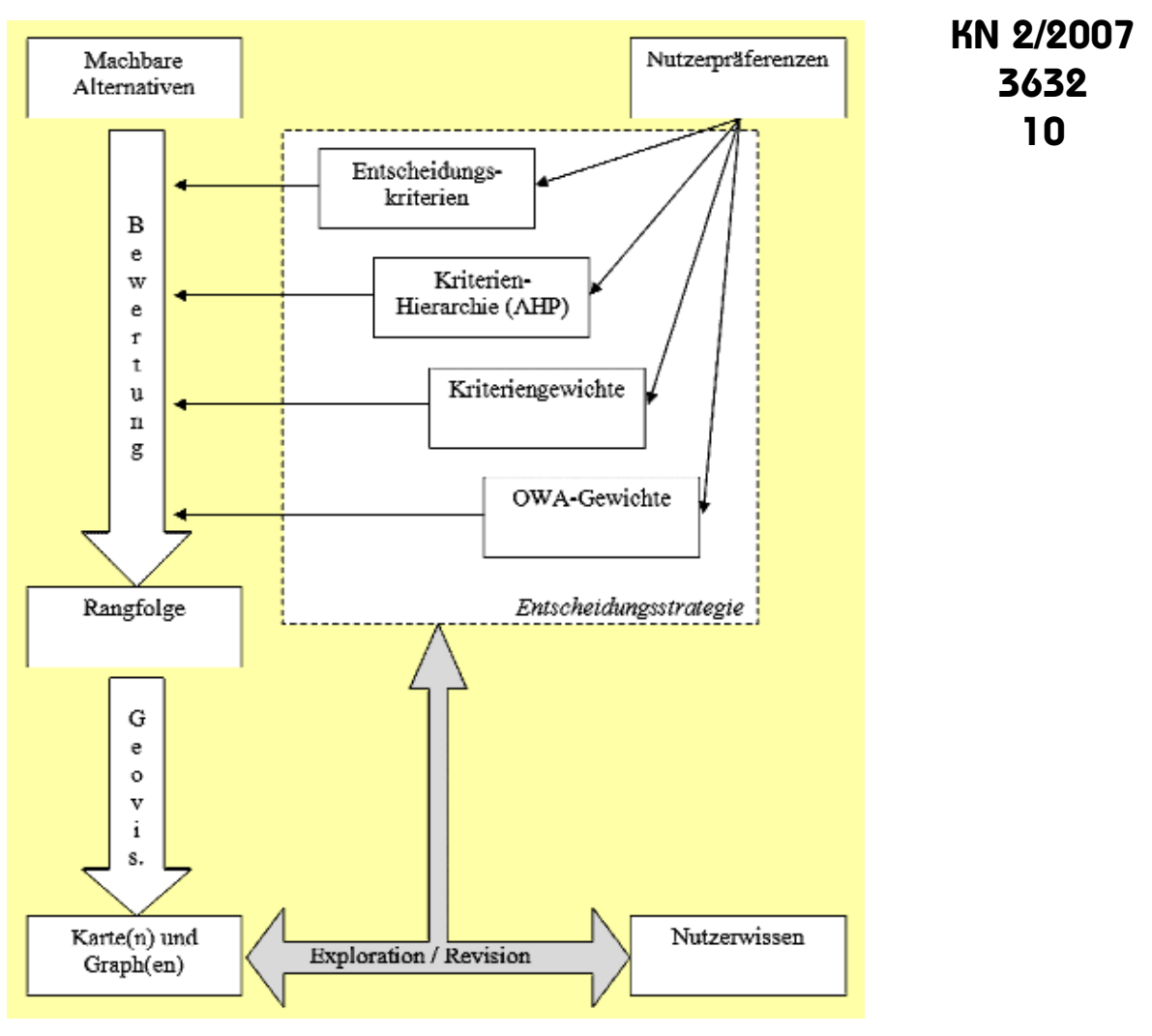

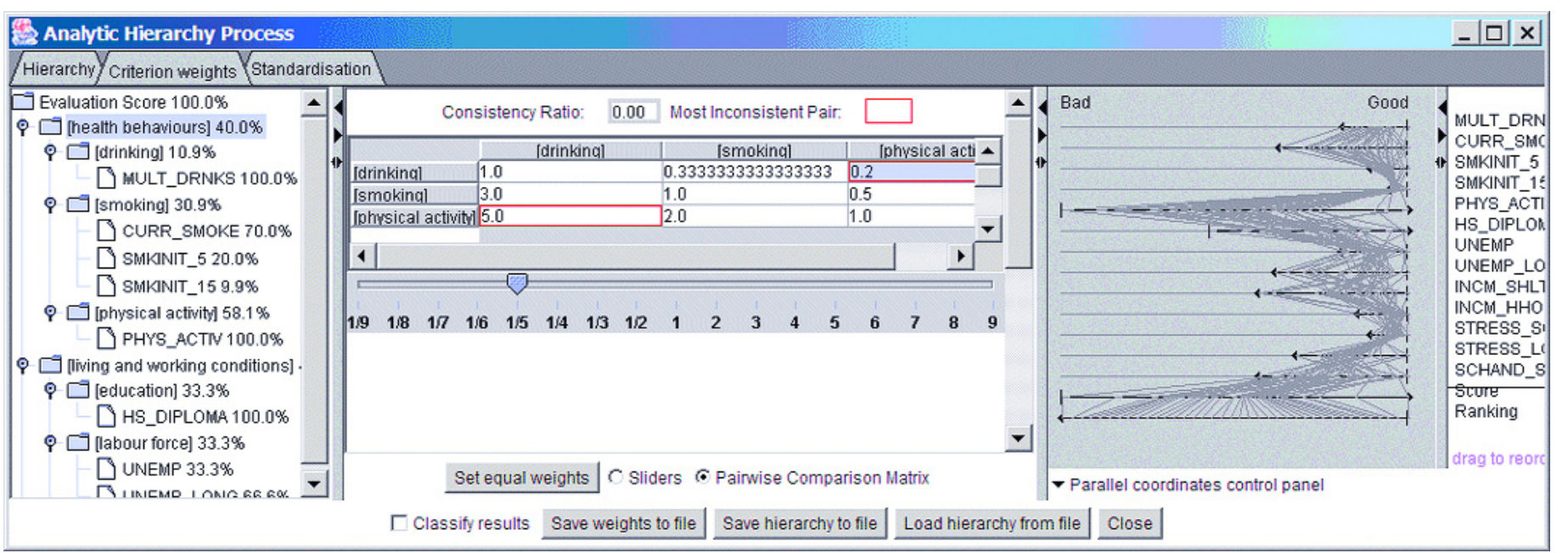
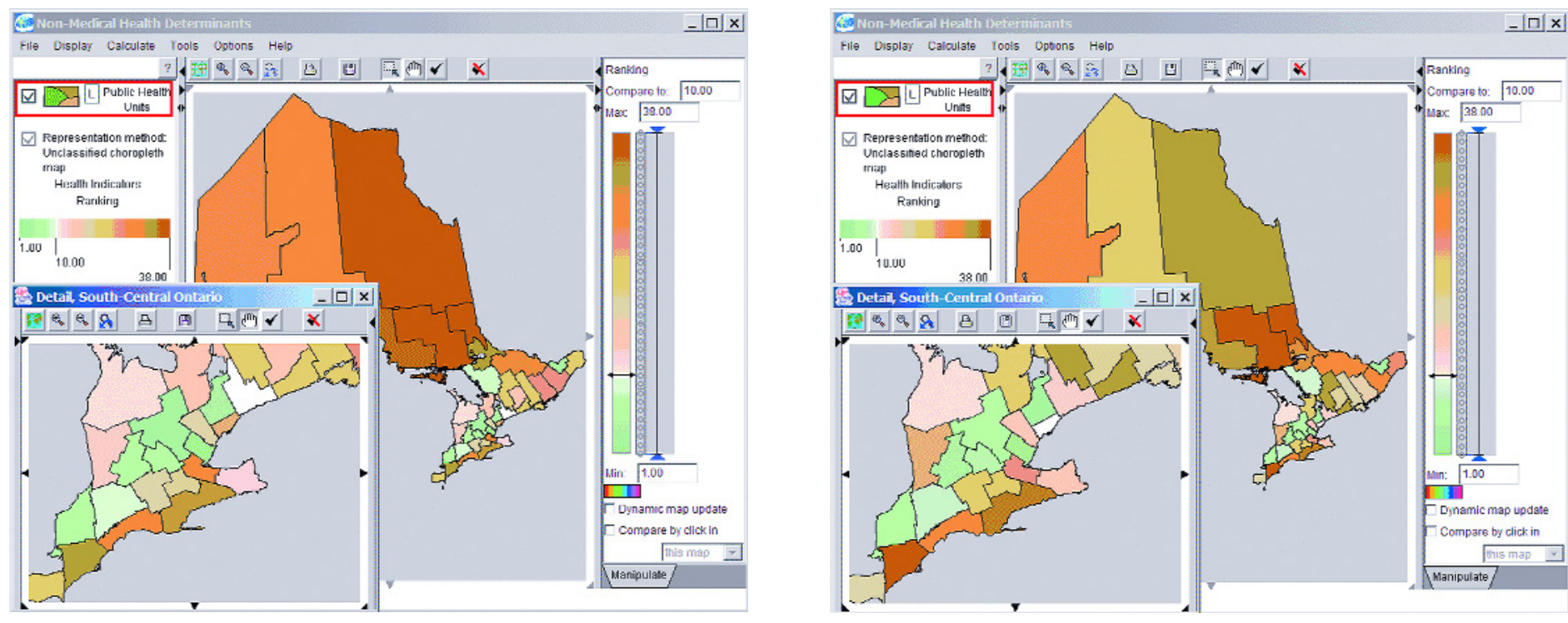


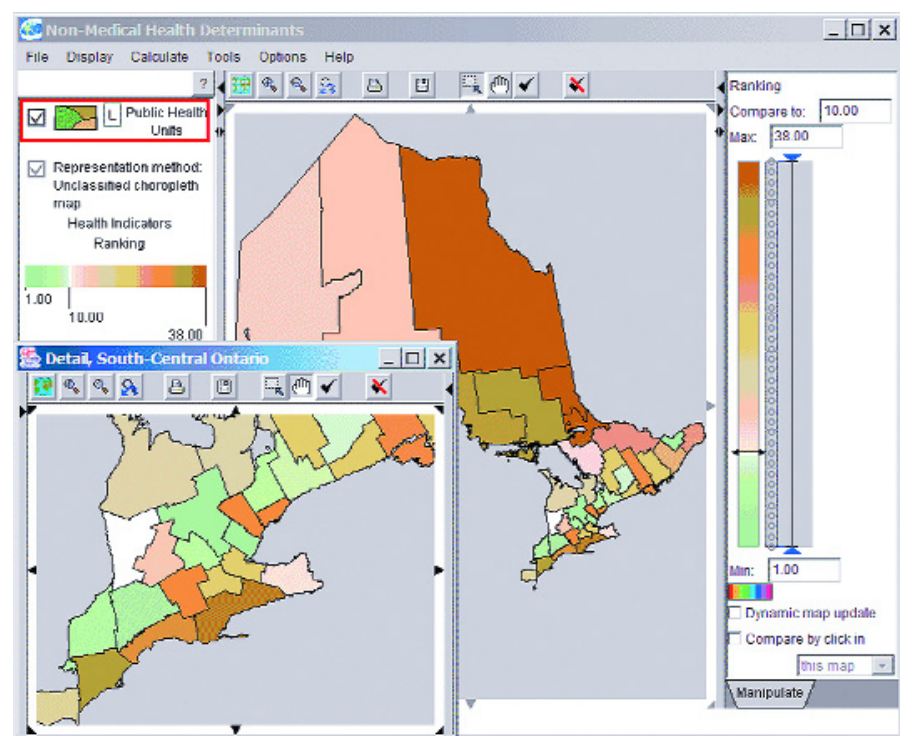

\section{KN 2/2007 \\ 3632}

11

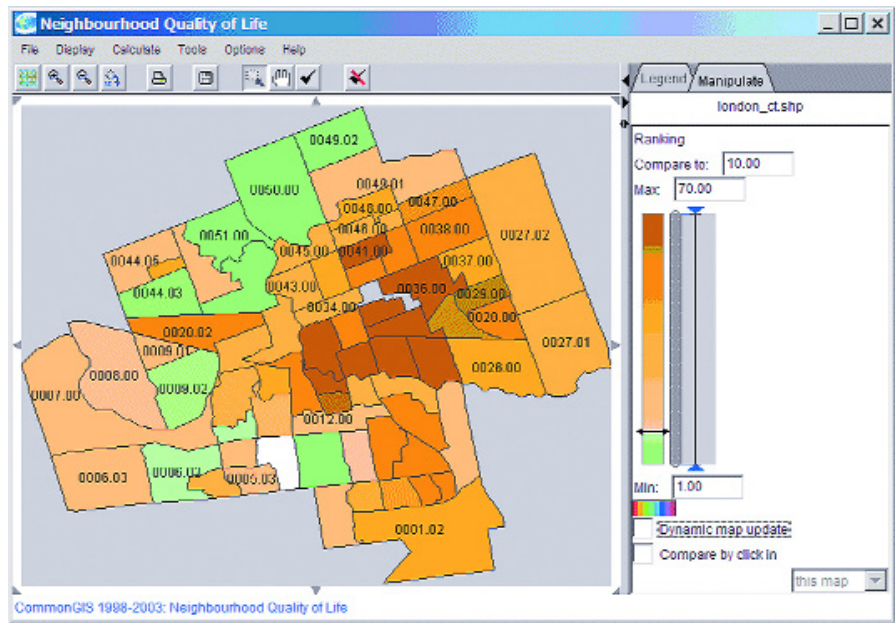

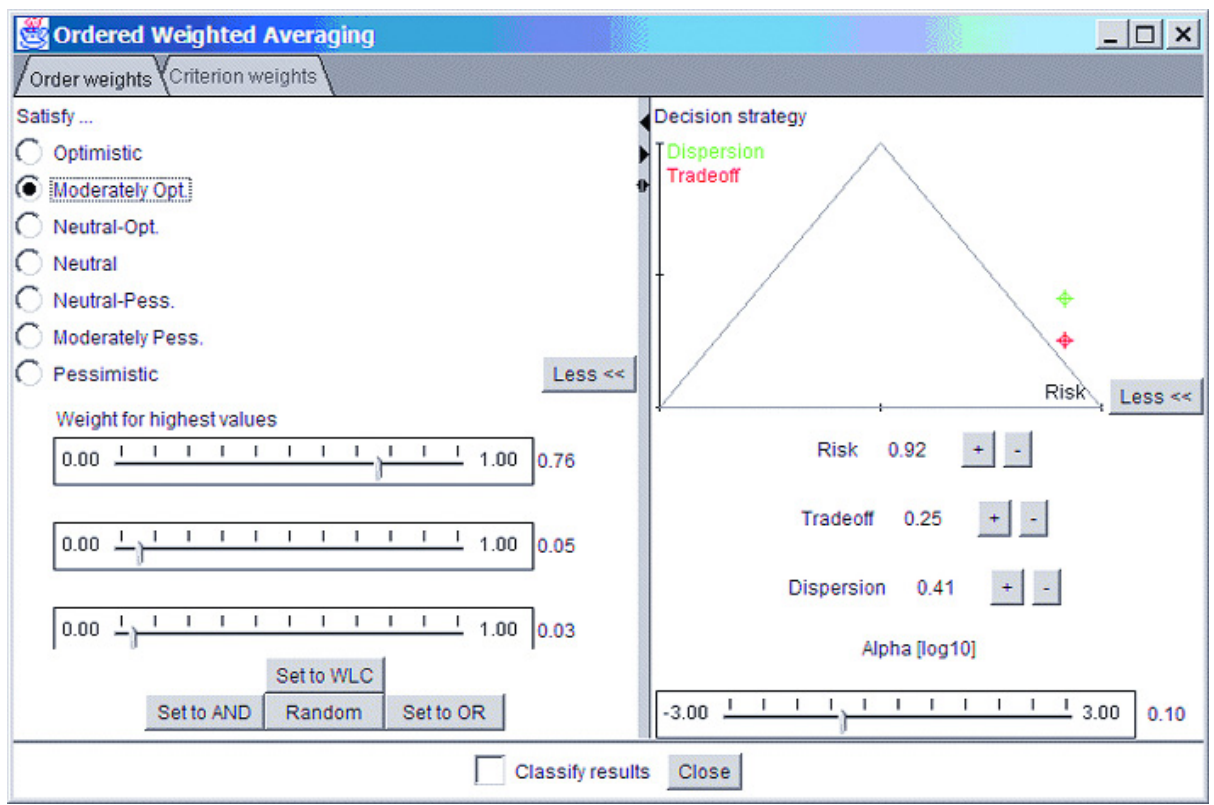
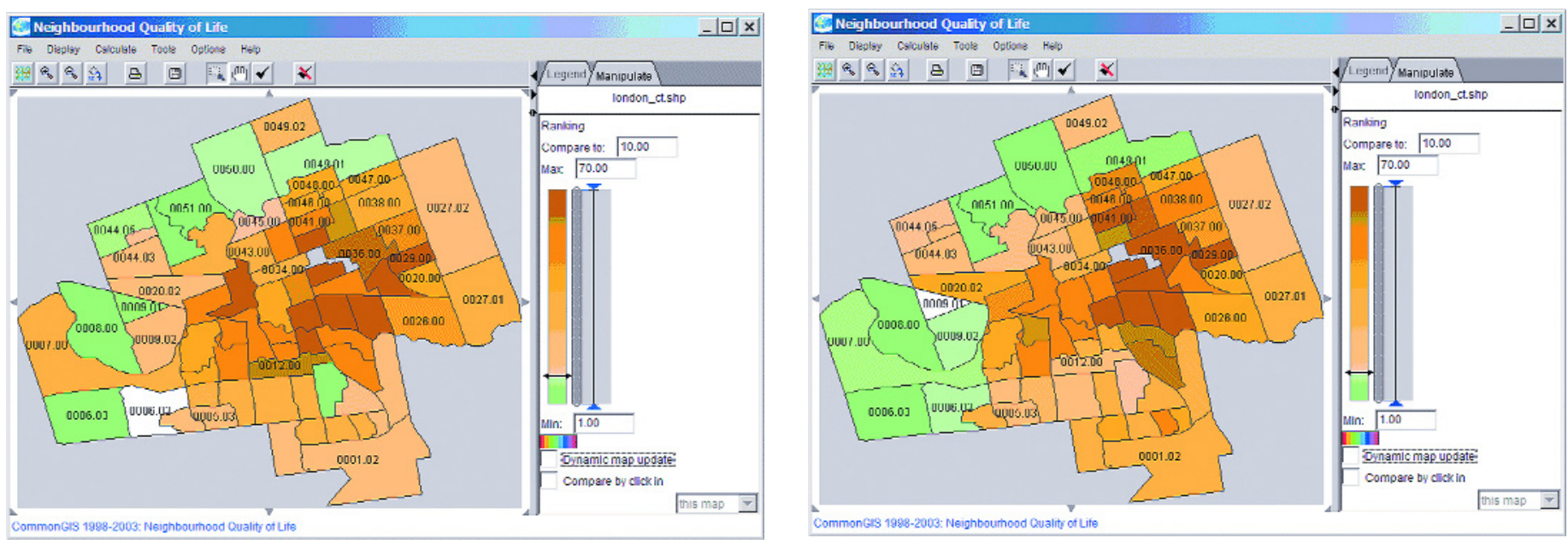


\begin{tabular}{|c|c|c|c|}
\hline $\begin{array}{l}\text { Hierarchie- } \\
\text { Ebene } 1\end{array}$ & $\begin{array}{l}\text { Hierarchie- } \\
\text { Ebene } 2\end{array}$ & Variablenname & Beschreibung \\
\hline \multirow[t]{5}{*}{$\begin{array}{l}\text { Gesundheits- } \\
\text { relevantes } \\
\text { Verhalten }\end{array}$} & $\begin{array}{l}\text { Trinkverhalten } \\
(\rightarrow>\text { zu } \\
\text { minimieren) }\end{array}$ & MULT_DRNKS & $\begin{array}{l}\text { Trinker ( } 5 \text { alkoholische Getränke, mind. } \\
12 \text { mal im Jahr, bez. auf nicht-abstinente } \\
\text { Bev. } \geq 12 \text { Jahre) }\end{array}$ \\
\hline & \multirow{3}{*}{$\begin{array}{l}\text { Rauchen } \\
(\rightarrow \text { min })\end{array}$} & CURR_SMOKE & Raucher (bez. auf Bev. $\geq 12$ Jahre) \\
\hline & & SMKINIT_5 & $\begin{array}{l}\text { Anteil der Raucher } \geq 12 \text { Jahre, die im Alter } \\
\text { zwischen } 5 \text { und } 14 \text { Jahren begonnen haben }\end{array}$ \\
\hline & & SMKINIT_15 & $\begin{array}{l}\text { Anteil der Raucher } 12 \text { Jahre, die im Alter } \\
\text { zwischen } 15 \text { und } 19 \text { Jahren begonnen haben }\end{array}$ \\
\hline & $\begin{array}{l}\text { Sport } \\
(\rightarrow>\max )\end{array}$ & PHYS_ACTIV & Anteil der physisch aktiven Bev. \\
\hline \multirow[t]{5}{*}{$\begin{array}{l}\text { Lebens- und } \\
\text { Arbeitsumstände }\end{array}$} & $\begin{array}{l}\text { Bildung } \\
(\rightarrow>\max )\end{array}$ & HS_DIPLOMA & $\begin{array}{l}\text { Anteil der Bev. zW. } 25 \text { und } 29 \text { Jahren mit } \\
\text { Highschool-Abschluss }\end{array}$ \\
\hline & \multirow[t]{2}{*}{$\begin{array}{l}\text { Arbeitsmarkt } \\
(\rightarrow>\min )\end{array}$} & UNEMP & $\begin{array}{l}\text { Arbeitslosenrate (bez. auf arbeitsfähige } \\
\text { Bev. } \geq 15 \text { Jahre) }\end{array}$ \\
\hline & & UNEMP_LONG & $\begin{array}{l}\text { Anteil der Langzeitarbeitslosen an der } \\
\text { arbeitsfähigen Bev. } \geq 15 \text { Jahre }\end{array}$ \\
\hline & \multirow[t]{2}{*}{$\begin{array}{l}\text { Einkommen } \\
(\rightarrow>\text { max })\end{array}$} & INCM_SHLTR & $\begin{array}{l}\text { Anteil der Haushalte, die } \geq 30 \% \text { ihres Ein- } \\
\text { kommens für Miete/Hypothek verwenden }\end{array}$ \\
\hline & & INCM_HHLD & $\begin{array}{l}\text { Anteil der Haushalte mit geringem Ein- } \\
\text { kommen nach ortsabhängiger Definition }\end{array}$ \\
\hline \multirow[t]{2}{*}{$\begin{array}{l}\text { Persönliche } \\
\text { Ressourcen }\end{array}$} & \multirow[t]{2}{*}{$\begin{array}{l}\text { Stress } \\
(\rightarrow>\min )\end{array}$} & STRESS_SOM & $\begin{array}{l}\text { Anteil der Bev. } \geq 18 \text { Jahre, die } \\
\text { chronischem Stress ausgesetzt ist }\end{array}$ \\
\hline & & STRESS_LOT & $\begin{array}{l}\text { Anteil der Bev. } \geq 18 \text { Jahre, die hohem } \\
\text { chronischem Stress ausgesetzt ist }\end{array}$ \\
\hline Umweltfaktoren & $\begin{array}{l}\text { Passivrauchen } \\
(\rightarrow \rightarrow \min )\end{array}$ & SCHAND_SMK & $\begin{array}{l}\text { Anteil der Nichtraucher, die im Haushalt } \\
\text { dem Passivrauchen ausgesetzt sind }\end{array}$ \\
\hline
\end{tabular}

\begin{tabular}{|c|c|}
\hline Variablenname & Beschreibung \\
\hline AVE_VAL $(->$ max) & Durchschnittlicher Wert (CAD) der Wohngebäude \\
\hline OLD_DWE (-> min) & Anteil der Wohneinheiten, die vor 1946 erbaut wurden \\
\hline NEW_DWE ( $\rightarrow$ max) & Anteil der Wohneinheiten, die nach 1980 erbaut wurden \\
\hline AVE_ROO (-> max) & Durchschnittliche Anzahl der Zimmer in Wohneinheiten \\
\hline NON_INC (-> min) & Anteil der Bevölkerung ( $\geq 15$ Jahre) ohne Arbeitseinkommen \\
\hline LOW_INC ( $\rightarrow$ min) & Anteil der Bevölkerung ( $\geq 15$ Jahre) mit geringem Einkommen \\
\hline GOV_PAY ( $\rightarrow$ min) & $\begin{array}{l}\text { Durchschnittlicher Anteil der Sozialleistungen am Gesamtein- } \\
\text { kommen der Haushalte }\end{array}$ \\
\hline UNE_RAT $(->\mathrm{min})$ & Arbeitslosenrate (Bev. $\geq 15$ Jahre) \\
\hline UNA_IND ( $\rightarrow$ min) & Single-Anteil der Bevölkerung ( $\geq 15$ Jahre) \\
\hline MOB_ONE (-> max) & $\begin{array}{l}\text { Anteil der Bevölkerung, der mindestens ein Jahr am gleichen Ort } \\
\text { wohnt }\end{array}$ \\
\hline LOW_EDU ( $\rightarrow>\min )$ & Anteil der Bevölkerung ( $\geq 15$ Jahre) ohne Schulabschluss \\
\hline HIG_EDU (-> max) & Anteil der Bevölkerung ( $\geq 15$ Jahre) mit Universitätsbildung \\
\hline NON_SCH (-> min) & Anteil der Bevölkerung (15 - 24 Jahre), der nicht in Ausbildung ist \\
\hline RES_BUR $(->\min )$ & Einbruchsquote in Wohnhäuser \\
\hline POP_DEN ( $\rightarrow$ min) & Bevölkerungsdichte \\
\hline
\end{tabular}

